\title{
Acupuncture for cancer pain: an evidence-based clinical practice guideline
}

\author{
Long Ge ${ }^{1,2,3,4 \dagger}$, Qi Wang ${ }^{1,2+}$, Yihan He $\mathrm{H}^{5,6,7+}$, Darong Wu ${ }^{5,6,7 \dagger}$, Qi Zhou ${ }^{8 \dagger}$, Nenggui Xu ${ }^{9 \dagger}$, Kehu Yang ${ }^{2,3,4,10 \dagger}$,
} Yaolong Chen ${ }^{3,4,8,10 \dagger}$, Anthony Lin Zhang ${ }^{1+}$, Haiqing Hua ${ }^{12 \dagger}$, Jinchang Huang ${ }^{13 \dagger}$, Ka-Kit Hui ${ }^{14 \dagger}$, Fanrong Liang ${ }^{15 \dagger}$, Linpeng Wang ${ }^{16 \dagger}$, Bin Xu ${ }^{17 \dagger}$, Yufei Yang ${ }^{18 \dagger}$, Weimin Zhang ${ }^{19 \dagger}$, Baixiao Zhao ${ }^{20 \dagger}$, Bing Zhu ${ }^{21 \dagger}$, Xinfeng Guo ${ }^{5,6,7^{*}}$, Charlie Changli Xue ${ }^{5,6,7,11^{*}}$ and Haibo Zhang ${ }^{5,6,7^{*}}$ on behalf of International Trustworthy traditional Chinese Medicine Recommendations (TCM Recs) Working Group

\begin{abstract}
Background: This study aims to develop an evidence-based clinical practice guideline of acupuncture in the treatment of patients with moderate and severe cancer pain.

Methods: The development of this guideline was triggered by a systematic review published in JAMA Oncology in 2020. We searched databases and websites for evidence on patient preferences and values, and other resources of using acupuncture for treatment of cancer pain. Recommendations were developed through a Delphi consensus of an international multidisciplinary panel including 13 western medicine oncologists, Chinese medicine/acupuncture clinical practitioners, and two patient representatives. The certainty of evidence, patient preferences and values, resources, and other factors were fully considered in formulating the recommendations. The Grading of Recommendations Assessment, Development, and Evaluation (GRADE) approach was employed to rate the certainty of evidence and the strength of recommendations.

Results: The guideline proposed three recommendations: (1) a strong recommendation for the treatment of acupuncture rather than no treatment to relieve pain in patients with moderate to severe cancer pain; (2) a weak recommendation for the combination treatments with acupuncture/acupressure to reduce pain intensity, decrease the opioid dose, and alleviate opioid-related side effects in moderate to severe cancer pain patients who are using analgesics; and (3) a strong recommendation for acupuncture in breast cancer patients to relieve their aromatase inhibitor-induced arthralgia.
\end{abstract}

\footnotetext{
*Correspondence: guoxinfeng@gzucm.edu; charlie.xue@rmit.edu.au; haibozh@gzucm.edu.cn

'tong Ge, Qi Wang and Yihan He are co-first authors

†Darong Wu, Qi Zhou, Nenggui Xu, Kehu Yang, Yaolong Chen, Anthony

Lin Zhang, Haiqing Hua, Jinchang Huang, Ka-Kit Hui, Fanrong Liang,

Linpeng Wang, Bin Xu, Yufei Yang, Weimin Zhang, Baixiao Zhao, Bing Zhu

contributed equally to this work

${ }^{5}$ Guangdong Provincial Hospital of Chinese Medicine, Guangzhou, China

${ }^{11}$ China-Australia International Research Centre for Chinese Medicine,

School of Health and Biomedical Sciences, RMIT University, Melbourne,

Victoria, Australia

Full list of author information is available at the end of the article
}

(c) The Author(s) 2022. Open Access This article is licensed under a Creative Commons Attribution 4.0 International License, which permits use, sharing, adaptation, distribution and reproduction in any medium or format, as long as you give appropriate credit to the original author(s) and the source, provide a link to the Creative Commons licence, and indicate if changes were made. The images or other third party material in this article are included in the article's Creative Commons licence, unless indicated otherwise in a credit line to the material. If material is not included in the article's Creative Commons licence and your intended use is not permitted by statutory regulation or exceeds the permitted use, you will need to obtain permission directly from the copyright holder. To view a copy of this licence, visit http://creativecommons.org/licenses/by/4.0/. The Creative Commons Public Domain Dedication waiver (http://creativeco mmons.org/publicdomain/zero/1.0/) applies to the data made available in this article, unless otherwise stated in a credit line to the data. 
Conclusion: This proposed guideline provides recommendations for the management of patients with cancer pain. The small sample sizes of evidence limit the strength of the recommendations and highlights the need for additional research.

Keywords: Acupuncture, Cancer pain, Practice guideline, Evidence-based practice

\section{Background}

Cancer is the second leading cause of death globally with an estimated mortality of 10.0 million in 2020 [ 1 , $2]$. Pain is one of the most common symptoms in cancer patients, in particular, over $70 \%$ of individuals with advanced cancer suffer from moderate to severe pain (Numerical rating scale, NRS $\geq 4$ ) [3, 4].

The World Health Organization (WHO) provides recommendations on pharmacologic and radiotherapeutic management of cancer pain, emphasizing the appropriate application of opioids [5]. However, the opioid crisis [6] has exacerbated the challenges of pain management and highlights a need for nonpharmacological treatments [7]. Acupuncture, as the most common method of traditional Chinese medicine in physical intervention, has been widely used for the control of chronic pain [8]. A recent systematic review and meta-analysis demonstrated that acupuncture was significantly associated with reduced cancer pain and can decrease the use of analgesics with moderate certainty of evidence [9]. Approximately one in 10 cancer survivors have used acupuncture in the United States [10]. Given the benefit, clinical urgency, easy public accessibility, relatively high social acceptance and low cost [11], it is necessary to develop a trustworthy guidance to guide clinical practice of acupuncture for cancer pain management.

Currently, several guidelines of the clinical management of cancer pain are available. Among them, the guidelines from European Oncology Nursing Society (EONS) and National Comprehensive Cancer Network (NCCN) $[12,13]$ suggest the use of acupuncture for managing cancer pain (Box 1). However, both of them do not provide the practical recommendations for acupuncture practice, which limits the application of acupuncture in clinical cancer pain management. Therefore, a specific guideline with detailed practical points is needed to promote the dissemination and implementation of acupuncture in clinical practice, particularly in the field of oncology [14].

The international Trustworthy traditional Chinese Medicine Recommendations (TCM Recs) Working Group, an international team aimed to produce rigorous evidence-based traditional Chinese medicine recommendations adhering to the trustworthy standards [15], has recruited an international multidisciplinary panel to develop this guideline. This guideline was mainly based on the systematic review published in JAMA Oncology [9]. Meanwhile, the working group also systematically reviewed the studies of patient preferences and values, and explored the rule of acupuncture point selection for cancer pain by literature review and Delphi method [16, 17].

This guideline is intended for all hospitals and community healthcare services, as well as Chinese medicine physicians, physicians of integrated traditional Chinese and western medicine, clinicians, rehabilitation physicians, acupuncturists, and related researchers. The target population is cancer patients with moderate to severe pain.

\section{Methods}

The design and development of this guideline are in accordance with the "World Health Organization Handbook for Guideline Development" [18]. The guideline was reported in accordance with the Reporting Items for Practice Guidelines in Healthcare (RIGHT) statement [19]. Appraisal of Guidelines for Research and Evaluation II (AGREE II) and Institute of Medicine (IOM) were consulted to ensure the quality of the guideline $[15,20]$. The development process of recommendations is summarized in Fig. 1.

\section{Panel constitution}

The guideline panel included representatives from the fields of western medicine, Chinese medicine/acupuncture, guideline and systematic review methodology,

Box 1 Current international guidelines on acupuncture for cancer pain

\begin{tabular}{ll}
\hline Guidelines & Recommendations \\
\hline European Oncology Nursing Society breakthrough cancer pain guide- & $\begin{array}{l}\text { Suggest acupuncture as a complementary strategy for cancer pain (unclear } \\
\text { strength of recommendation) }\end{array}$ \\
lines, 2014 & Suggest acupuncture as a physical intervention for cancer pain (category 2A) \\
NCCN guideline: Adult Cancer Pain, Version 2.2021, 2021 &
\end{tabular}




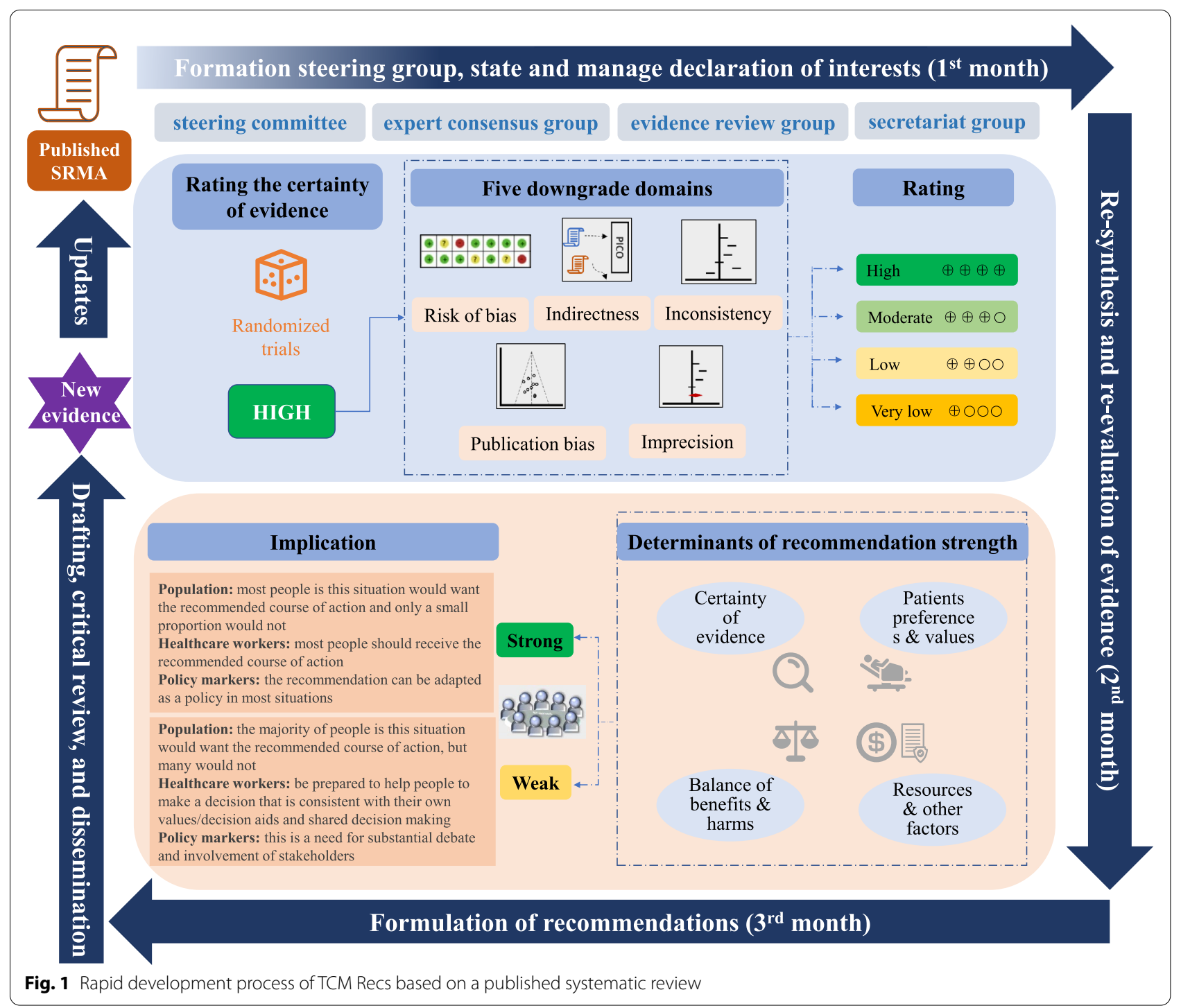

as well as two patients. The working group consisted of four subgroups including a steering committee, an expert consensus group, an evidence review group, and an academic secretariat group. The professional fields of the steering committee and the expert consensus group are shown in Additional file 1: Appendix S1. All members of the panel completed a disclosure form, which required disclosure of financial and other interests, including relationships with commercial entities. None of the panel members stated any financial conflict of interest. The members who had academic conflicts of interest (the core authors of JAMA Oncology systematic review) did not participate in voting on the recommendations (Additional file 1: Appendix S2).

\section{Identification of clinical questions}

Based on the systematic review published in JAMA Oncology [9], in accordance with the Patients, Intervention, Control and Outcomes (PICO) framework, the secretariat group drafted clinical questions. Then, the steering committee conducted an online face-to-face discussion through Microsoft Team meeting, and initially identified five clinical questions: (1) with regard to pain alleviation for cancer patients, what are the effects of acupuncture treatment? (2) For cancer patients using analgesics for pain control, what are the effects of acupuncture/acupressure when combined with analgesics? (3) What are the differences of different acupuncture methods in pain alleviation for cancer patients? (4) What are the acupoints selection, manipulation and course of 
acupuncture /acupressure treatment for cancer pain? (5) With regard to breast cancer patients with aromatase inhibitor-induced arthralgia, what are the effects of acupuncture treatment? We invited all experts of the consensus group to vote the rationality those five clinical questions. Finally, we decided to describe the third and the fourth clinical questions under the description of the first clinical question as the recommendation statement.

\section{Synthesis and evaluation of evidence}

The evidence review group closely collaborated with the core authors of the JAMA Oncology systematic review. Using the studies included in the systematic review, we re-performed data synthesis and certainty of evidence assessment based on the clinical questions. Data synthesis was performed using Review Manager software (RevMan, Version 5.3, Copenhagen: The Nordic Cochrane Centre, The Cochrane Collaboration, 2014). We rated the certainty of evidence for each outcome using GRADE approach [21, 22], which classifies evidence as high, moderate, low, or very low certainty (Additional file 1: Appendix S3). The starting point for certainty for randomized controlled trials (RCTs) is high, but can be rated down based on serious limitations in risk of bias, imprecision, inconsistency (heterogeneity), indirectness, and publication bias. We used minimally important differences (MIDs) to assess the imprecision of summary effect estimates based on the Initiative on Methods, Measurement, and Pain Assessment in Clinical Trials (IMMPACT) recommendations [23].

We adopted literature review and Delphi method to explore the rule of acupuncture point selection for cancer pain [24]. We searched Chinese and English databases to include clinical studies on the treatment using acupuncture for cancer pain. We included 28 studies and extracted acupoints and selection principles adopted in the included clinical studies. The questionnaire was designed according to the literature review results, and two rounds of Delphi survey were conducted among 34 Chinese and international experts in the field of acupuncture and cancer.

\section{Patient preferences and values}

We searched eight databases from the inception to June 2020 to include primary quantitative (e.g. cross-sectional surveys), qualitative (e.g. interviews, focus groups), and mixed method studies on the preferences and values of patients with cancer pain. Quantitative studies used an adapted version of the GRADE approach to assess risk of bias of studies [25] and qualitative studies used the Critical Appraisal Skills Programme qualitative research checklist [26]. Teams of two independently performed titles and abstracts screening, full-text review, data extraction, and risk of bias assessment. Any conflict was resolved by consensus. Details on search and screening can be found in Additional file 1: Appendix S4. We finally included 8 studies involved 2505 patients with cancer pain. We did not find any published evidence of acupressure addressing patient values and preferences. The results were analyzed and taken into account when developing evidence-based recommendations. Meanwhile, we also included two patients, who have used acupuncture for the treatment of their cancer pain, to participate in voting the recommendations.

\section{Formulation of recommendations}

We used GRADE approach to formulate the recommendations, and categorized the strength of recommendations as "strong" and "weak" (sometime guidelines may use terms such as "conditional" or "discretionary" instead of weak), by comprehensively considering the balance of benefits and harms, the certainty of evidence, the patient preferences and values, resources, and other factors [21, 22]. We searched PubMed and Chinese National Knowledge Infrastructure databases to identify studies on the accessibility, acceptability, and policy support for acupuncture or acupressure.

We used Delphi method to reach consensus for all recommendations. The consensus was reached when more than $70 \%$ experts agreed with the strength and direction of recommendation. We obtained the feedback regarding the recommendation from 2 patients and 12 experts (one expert did not vote). The acupuncturist first explained to patients the content that needs consensus, and then the patients independently complete the consensus voting on recommendations.

In the first round of Delphi survey, all recommendations reached a consensus. Thirteen of 14 experts voted for recommending the treatment of acupuncture to relieve pain and suggesting a combination treatment of acupuncture/acupressure and analgesics to relieve pain and reduce opioid dose in patients with cancer pain. Ten of 14 experts voted for suggesting the treatment of acupuncture rather than no treatment to relieve pain in breast cancer patients with aromatase inhibitor-induced arthralgia. The consensus was also reached for the acupuncture point selection, manipulation and course. The details are shown in Additional file 1: Appendix S5.

\section{Drafting, critical review, and dissemination}

The guideline was drafted by the academic secretariat. After an internal review, a document was developed for open discussion. After receiving feedbacks from experts, the guideline was finalized by another round of peer review facilitated by the journal editor. The guideline will be disseminated by peer-reviewed academic journals, 
presented at conferences, and promoted in public media outlets such as WeChat and Facebook.

\section{Updates}

As new evidence is published, the TCM Recommendations group will assess the new evidence and make a judgment on the extent that it would be expected to alter the recommendation. Updated guideline will follow the Checklist for the Reporting of Updated Guidelines (CheckUp) [27].

\section{Recommendations}

The recommendations are summarized in Fig. 2.

Clinical question 1: With regard to pain alleviation for cancer patients, what are the effects of acupuncture treatment?

Recommendation 1

We recommend the treatment of acupuncture rather than no treatment to relieve pain in patients with moderate to severe cancer pain (strong recommendation, moderate certainty evidence).

\section{Recommendation statement}

1.1 There was no significant difference in relieving cancer pain using different acupuncture techniques (manual acupuncture, electroacupuncture and auricular acupuncture).

1.2 Personalized acupoint selection is suggested. The main acupoints are Hegu (LI4), Taichong (LR3), Ashi-point, Zusanli (ST36), Sanyinjiao (SP6), and Yanglingquan (GB34).

1.3 Manipulation

Manual acupuncture: The needling consisted of swabbing all acupoints with alcohol. Needles were inserted to a proper depth as determined by standard point locations, and a "De qi" sensation was obtained at all standardized full-body acupuncture points. During the time of acupuncture, the acupuncturist returned to stimulate needles once, to re-elicit the "De qi" sensation. The course is recommended twice a week, 30 min each time for 6 weeks. Due to limited available evidence, it is recommended to consider specific clinical situation in clinical practice.

Electroacupuncture: Two pairs of electrodes were connected at the needles adjacent to the site of pain with two frequencies (hertz) of electro-stimulation provided by a TENS (Transcutaneous Electric Nerve Stimulation) unit. The course is recommended twice a week for 2 weeks, and then weekly for 6 weeks. The needles were left in place for 30 min each time. Due to limited available evidence, it is recommended to consider specific clinical situation in clinical practice.

Auricular acupuncture: Use sterile, disposable, semi-permanent needles for 40 min per session, with the needle remaining in the auricle for 3 days. Due to limited available evidence, it is recommended to consider specific clinical situation in the clinical practice.

\section{Benefits and harms}

Eight randomized controlled trials (RCTs) [28-35], with a total of 530 patients with moderate to severe pain, compared the efficacy of acupuncture and sham acupuncture or wait-list. The results showed that acupuncture could effectively alleviate the intensity of cancer pain (real acupuncture vs. sham acupuncture [28-34], $\mathrm{n}=398$, NRS score change: $\mathrm{MD}=-1.39$ points; $95 \% \mathrm{CI}-2.15$ to -0.63 ; real acupuncture vs. wait-list control [31, 33, $35], \mathrm{n}=255$, NRS score change: $\mathrm{MD}=-1.63$ points; $95 \%$ CI -2.14 to -1.13 ) (see Additional file 1: Appendix S6: Tables S1, S2 and Fig. S1 for summary). No serious treatment-related adverse events were observed. The certainty for evidence was moderate.

Three types of acupuncture including manual acupuncture, electroacupuncture, and auricular acupuncture were identified in included RCTs (Additional file 1: Appendix S6: Tables S3-S5 and Figs. S2-S4). We conducted subgroup analysis based on the types of acupuncture and no statistically significant subgroup effect was found (test of interaction: $P=0.66$ ), indicating that there was no significant difference in relieving cancer pain among different types of acupuncture (Additional file 1: Appendix S6: Fig. S5).

\section{Clinical question 2: For cancer patients using analgesics for pain control, what are the effects of acupuncture/ acupressure when combined with analgesics? Recommendation 2}

We suggest a combination treatment with acupuncture/ acupressure to reduce pain intensity, decrease opioid dose, and alleviate opioid-related side effects in moderate to severe cancer pain patients who are using analgesics (weak recommendation, low certainty evidence).

\section{Recommendation statement}

Acupoint selection and manipulation of acupuncture treatment could refer to the recommendation 1. For acupressure, we suggest Shenmen (HT7), Subcortex, and Sympathetic Nerve acupoints. The manipulation is: the patient takes the supine position, and the acupoint skin 

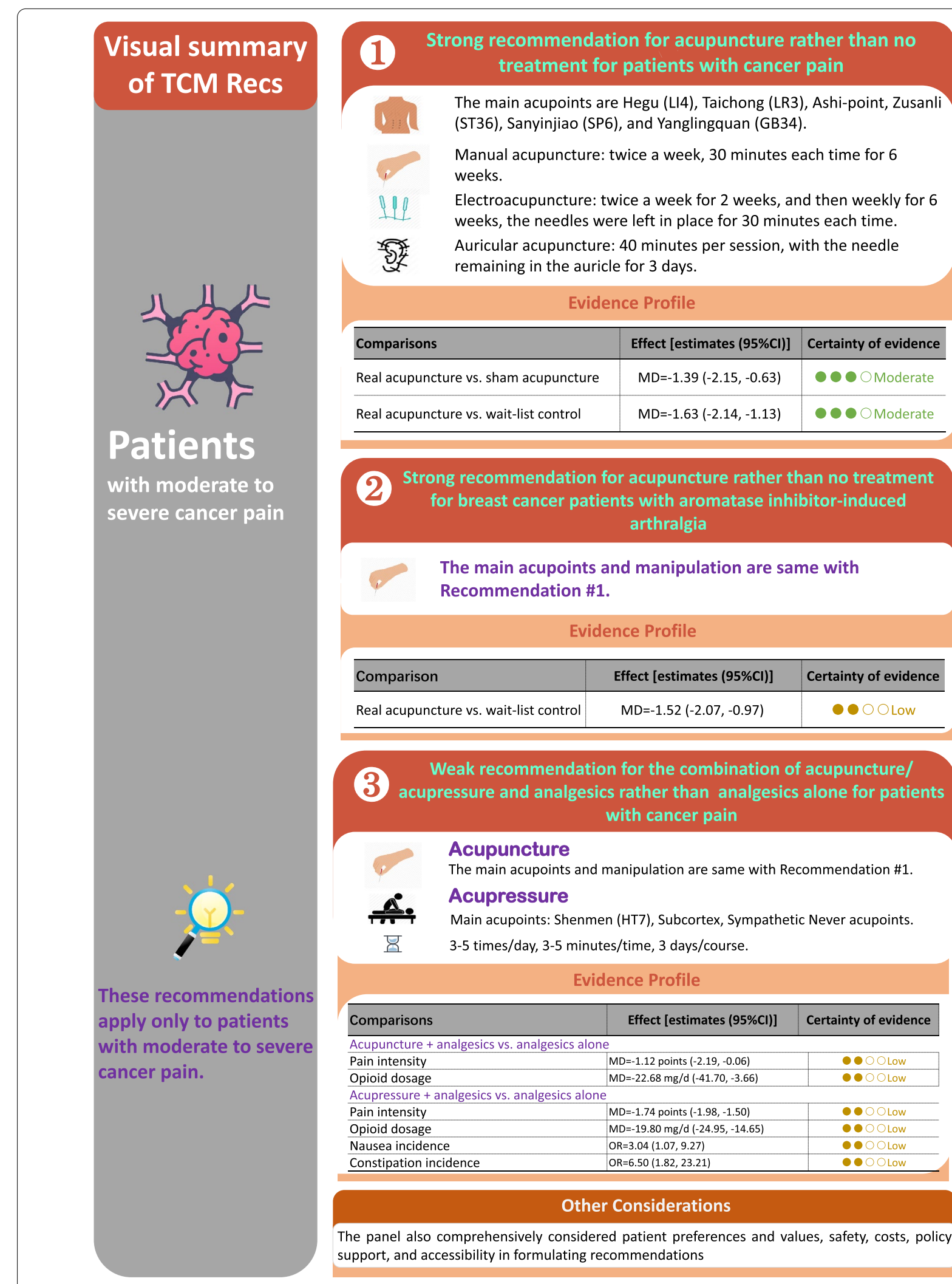

The main acupoints and manipulation are same with
Recommendation \#1.
Evidence Profile
\begin{tabular}{|l|c|c}
\hline Comparison & Effect [estimates $(95 \% \mathrm{Cl})]$ & Certainty of evidence \\
\hline Real acupuncture vs. wait-list control & $\mathrm{MD}=-1.52(-2.07,-0.97)$ & $\bigcirc$ \\
\hline
\end{tabular}

\section{Weak recommendation for the combination of acupuncture/ \\ 3 acupressure and analgesics rather than analgesics alone for patients with cancer pain}

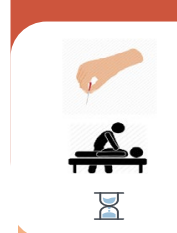

\section{Acupuncture}

The main acupoints and manipulation are same with Recommendation \#1.

\section{Acupressure}

₹ $\quad 3-5$ times/day, 3-5 minutes/time, 3 days/course.

\begin{tabular}{|c|c|c|}
\hline \multicolumn{3}{|c|}{ Evidence Profile } \\
\hline Comparisons & Effect [estimates $(95 \% \mathrm{CI})]$ & Certainty of evidence \\
\hline \multicolumn{3}{|c|}{ Acupuncture + analgesics vs. analgesics alone } \\
\hline Pain intensity & $M D=-1.12$ points $(-2.19,-0.06)$ & 000 Low \\
\hline Opioid dosage & $M D=-22.68 \mathrm{mg} / \mathrm{d}(-41.70,-3.66)$ & $\bullet \bullet \bigcirc \mathrm{Low}$ \\
\hline \multicolumn{3}{|c|}{ Acupressure + analgesics vs. analgesics alone } \\
\hline Pain intensity & $M D=-1.74$ points $(-1.98,-1.50)$ & - $\bigcirc \circ$ Low \\
\hline Opioid dosage & $M D=-19.80 \mathrm{mg} / \mathrm{d}(-24.95,-14.65)$ & 000 Low \\
\hline Nausea incidence & OR=3.04 $(1.07,9.27)$ & 000 Low \\
\hline Constipation incidence & $\mathrm{OR}=6.50(1.82,23.21)$ & 000 Low \\
\hline
\end{tabular}

\section{Other Considerations}

The panel also comprehensively considered patient preferences and values, safety, costs, policy support, and accessibility in formulating recommendations

Fig. 2 Visual summary of recommendations 
was routinely disinfected with $75 \%$ alcohol. A small piece of tape sticks the cowherb seeds tightly on the acupoints and replaces them daily. Use the index finger and thumb to twist and press the ear points on both sides of the ears, starting from light to heavy. It is suggested that this procedure be performed 3 to 5 times a day in the morning, afternoon, and evening, each time for 3 to $5 \mathrm{~min}$. The pressing force should be an acid bilge feeling in the local point, and 3 days as one course. Due to limited available evidence, the procedure should consider specific clinical practice.

\section{Benefits and harms}

Three RCTs addressed 224 patients [36-38], and compared a combination of acupuncture and analgesic drugs to analgesic drugs alone. The results suggested that acupuncture combined with analgesics could effectively relieve the intensity of pain (3 studies [36-38], $\mathrm{n}=224$, NRS score change: $\mathrm{MD}=-1.12$ points; $95 \% \mathrm{CI}-2.19$ to -0.06 ), reduce the use of opioid (1 study [38], $\mathrm{n}=60$, dose changes: $\mathrm{MD}=-22.68 \mathrm{mg} / \mathrm{d} ; 95 \% \mathrm{CI}-41.70$ to -3.66) (Additional file 1: Appendix S6: Fig. S6).

The systematic review included 3 RCTs with 166 patients with severe cancer pain [39-41] showed that acupressure combined with analgesics could effectively relieve the intensity of pain ( 3 studies, $n=166$, NRS score change: $\mathrm{MD}=-1.74$ points; $95 \% \mathrm{CI}-1.98$ to -1.50 ) and reduce the use of opioid ( 1 study [40], $n=46$, the dose change: $\mathrm{MD}=-19.80 \mathrm{mg} / \mathrm{d} ; 95 \% \mathrm{CI}-24.95$ to -14.65) (Additional file 1: Appendix S6: Fig. S7).

The certainty of evidence for all outcomes above were low (Additional file 1: Appendix S6: Tables S6, S7).

No serious acupuncture-related adverse events were observed. One RCT [39] involving 60 patients with moderate to severe cancer pain showed that with low certainty of evidence, acupressure combined with analgesic could reduce the incidence of nausea and constipation (nausea: $\mathrm{OR}=3.04 ; 95 \% \mathrm{CI} 1.07$ to 9.27; constipation: $\mathrm{OR}=6.50 ; 95 \%$ CI 1.82 to 23.21 ).

\section{Clinical question 3: With regard to breast cancer patients with aromatase inhibitor-induced arthralgia (AllA), what are the effects of acupuncture treatment? \\ Recommendation 3}

We recommend the treatment of acupuncture rather than no treatment to relieve pain in breast cancer patients with aromatase inhibitor-induced arthralgia (strong recommendation, low certainty evidence).

\section{Recommendation statement}

Acupoint selection and procedure of acupuncture treatment could refer to the recommendation 1 .

\section{Benefits and harms}

The systematic review included 2 RCTs involving 197 patients with moderate to severe cancer pain [31,33]. The results showed that acupuncture could effectively alleviate the intensity of joint pain caused by aromatase inhibitors (acupuncture vs. wait-list control, NRS score change: $\mathrm{MD}=-1.52$ points; $95 \% \mathrm{CI}-2.07$ to -0.97 ; low certainty) (see Additional file 1: Appendix S6: Table S8 and Fig. S8). No serious treatment-related adverse events of acupuncture were observed.

\section{Preference and values}

Eight [42-49] cross-sectional surveys, that involved 2505 American and Swedish patients, investigated patient's preference, attitude and barriers. Additional file 1: Appendix S4 presents the details of the study of patient preferences and values, that included search strategies, study screening, summary of included studies, and risk of bias assessment.

Most of cancer patients (ranged from 79 to 97\%) thought that acupuncture was worthwhile, important, or effective. Around one third of cancer pain patients (ranged 27\% to $42 \%$ ) preferred acupuncture over medication for pain management. For the patients who received acupuncture treatment, most of them (ranged from 70 to $87 \%$ ) stated that the course of acupuncture met their expectations; and most of them (ranged 90\% to 100\%) were willing to receive acupuncture treatment again and were willing to recommend acupuncture to others. The main attitudinal barriers to receive acupuncture treatment included the lack of knowledge of acupuncture, expensive, and needling pain.

\section{Resources and other considerations Safety}

The evidence of from the JAMA Oncology's systematic review showed that the adverse events consisted predominantly the skin and subcutaneous tissue disorder or slight pain from the application of treatment to the skin. No dropouts were attributed to adverse effects associated with acupuncture treatment [9]. A study involving 454,920 patients who used acupuncture to treat pain showed that $8 \%$ patients experienced mild adverse events, severe side effects occurred in 13 patients $(0.003 \%)$ [50]. The most frequent mild adverse events included needling pain, hematoma, and bleeding at the point of needle insertion. Severe side reactions were pneumothorax, acute hyper- or hypotensive crisis, erisypelis, and asthma attack [50]. A systematic review indicated that acupuncture performed by trained practitioners using clean needle techniques was a generally safe procedure [51]. A review also showed that due to the low risks of using 
acupuncture, it could be successfully used for symptom management of cancer patients [52].

\section{Costs}

A systematic review included 8 studies on cost-utility and cost effectiveness of acupuncture for chronic pain showed that the cost per quality-adjusted life-year gained was below the thresholds used by the UK National Institute for Health and Clinical Excellence for "willingness to pay" [53]. A study on the out-of-pocket costs incurred from acupuncture services investigated acupuncture prices of 723 clinics throughout 39 metropolitan regions in the U.S., found that the median cost for a first-time acupuncture visit was $\$ 112$, and $\$ 80$ for the follow-up visits [54].

\section{Policy}

Acupuncture is receiving increasing policy support around the world, especially in the area of health insurance.

In USA, acupuncture has been recommended as a non-pharmacologic therapy of the most evidence-based and immediately available choice by the Food and Drug Administration (FDA) as well as the National Academies of Sciences, Engineering, and Medicine (NASEM) in coping with the opioid crisis [55]. Acupuncture was included for the first time in Canada by the province of British Columbia's Medical Services Plan (MSP) for those who are covered under premium assistance in 2008. This includes patients who are eligible $\mathrm{BC}$ residents who have a combined family income of $\$ 28,000$ or less. Patients have coverage for $\$ 23$ per visit per year for a maximum combination of 10 sessions from registered acupuncturists and related practitioners [56].

In Australia, the practice of acupuncture has been nationally regulated since 2012. Acupuncture services can be reimbursed by private health funds, and when practiced by medical practitioners, covered by the Australia's Medicare Benefits Schedule [57]. The Medicare benefit is divided into four levels, including Level A (obvious and straightforward cases and necessary examination, fee: $\$ 21.65$, benefit $75 \%$ ), Level B (a consultation lasting less than $20 \mathrm{~min}$ for cases that are not obvious or straightforward in relation to one or more health related issues, fee: $\$ 37.05$, benefit $100 \%$ ), Level C (a consultation lasting at least $20 \mathrm{~min}$ for cases in relation to one or more health related issues, fee: $\$ 71.70$, benefit $100 \%$ ), and Level $\mathrm{D}$ (a consultation lasting at least $40 \mathrm{~min}$ for cases in relation to one or more health related issues, fee: $\$ 105.55$, benefit 100\%).

Chinese medicine in Switzerland is supported by basic and additional medical insurance, which is one of the countries with the greatest support for acupuncture programs [58]. The cost of an acupuncture treatment is about 100-150 Swiss francs, and most patients can be reimbursed $80 \%$ from the insurance company. If the doctor performing the treatment is a qualified western doctor, the reimbursement amount will be even greater [59].

In China, National Administration of Traditional Chinese Medicine issued a document, including acupuncture and moxibustion, therapeutic massage, scrapping, cupping and other TCM non-drug diagnosis and treatment technologies into the new rural cooperative medical insurance, $60 \%$ reimbursement for township hospitals, $40 \%$ for secondary hospitals, and 30\% for tertiary hospitals [60].

\section{Accessibility}

Acupuncture has been widely accessible in many countries. The American Academy of Medical Acupuncture (AAMA) has more than 1300 medical doctors trained to offer acupuncture services, and has approved nine programs for medical doctor certification in acupuncture $[55,61]$. With the implementation of the Affordable Care Act (ACA), more than 54 million Americans have received acupuncture coverage under the Essential Healthcare Benefit [62]. While the ACA did stipulate acupuncture is a covered modality, the implementation defers to states, which is influenced by both the inertia of the conventional medical establishment and insurance companies' policies. A survey in 2015 found that the number of licensed acupuncturists in the US was 34,481, up 52.09\% from 2004; there were 62 master degree and 10 doctoral degree programs or schools nationwide, and at the same time, 44 states and the District of Columbia that had acupuncture practice laws in place.

In Australia, there are 4921 registered Chinese medicine practitioners in 2020, and $97.9 \%$ of them registered as an acupuncturist [63]. In the UK, the majority of acupuncture is performed by health professionals, including doctors, nurses, physiotherapists, midwives and nonmedically trained practitioners. All acupuncturists must be members of the appropriate statutory regulatory body, such as General Medical Council for doctors and comply with their standards and codes of conduct. More than a decade ago, the British issued a policy document, as a guideline, which set out a basic, minimum standard of care for acupuncture for cancer pain [64-66]. The British Acupuncture Council (BAcC) has a membership of over 3000 professionally qualified acupuncturists, which is the UK's largest professional or self-regulatory body for the practice of traditional acupuncture. Each year 2.3 million traditional acupuncture treatments are carried out in the UK, making it one of the most popular complementary therapies [67]. 
On the other hand, with the development of integrative oncology around the world [68-70], acupuncture has been adopted as part of routine clinical care in the integrative oncology setting [71, 72]. With the wide accessibility of acupuncture, it will continue to play an important role in the field of oncology.

\section{Discussion}

Cancer pain is one of the most common symptoms in cancer patients, especially in advanced cancer patients. However, there were limited effective strategies for pain management for most patients with moderate to severe cancer pain, which significantly reduce the quality of life of patients. Acupuncture can be effective in the management of not only pain, but also symptoms such as insomnia, anxiety, and joint pain [73]. The non-drug treatment and acupuncture therapy were demonstrated to be effective based on available clinical evidence, which offered new avenues for options of managing cancer pain.

This is the first evidence-based guideline for acupuncture in the treatment of cancer pain, and is expected to improve quality of life and care quality for moderate and severe cancer patients. The development of this guideline aims to standardize the clinical practice of acupuncture and ensure its safe and effective application. These guideline recommendations were developed with some strength. Firstly, the evidence was mainly based on a high-quality systematic review published in JAMA Oncology in 2020. We conducted meta-analyses to adopt different clinical questions and rated the certainty of evidence with GRADE approach. We focused on the breast cancer patients with aromatase inhibitor-induced arthralgia because of sufficient evidence. Secondly, we recruited 12 international multidisciplinary experts to perform Delphi consensus and formulated guideline recommendations by comprehensively considering the magnitude of effect measures, certainty of evidence, patient preferences and values, cost, policy, and accessibility. Thirdly, we considered patient preferences and values based on a systematical review and 2 patient representatives.

However, there were some limitations in this study. Firstly, most of the evidence was of low certainty, and therefore we made weak recommendations. Second, JAMA Oncology systematic review searched evidence up to March 31, 2019. We did not update this systematic review because only one additional relevant study [74] was published after their search and could not change the conclusion. Third, the overall evidence is insufficient to recommend acupuncture/acupressure for a specific type of cancer except breast cancer.

Future research should prioritize to address: (1) if the efficacy of acupuncture varies in alleviating cancer pain for different types of cancer; (2) the efficacy of acupressure to relieve cancer pain; (3) the patient preferences, values and attitude barriers of acupuncture for cancer pain from different countries.

\section{Conclusion}

In conclusion, we proposed the first evidence-based guideline recommendations for the management of cancer patients with moderate to severe pain. This guideline will benefit patients with cancer pain using acupuncture treatment. Dissemination and implementation of this guideline are integral components of our goals to address the management of cancer pain. The lack of high-quality evidence limits the strength of the recommendations and highlights the need for additional research.

\section{Abbreviations}

NRS: Numerical Rating Scale; WHO: World Health Organization; TCM: Traditional Chinese Medicine; GRADE: The Grading of Recommendations Assessment, Development and Evaluation; RIGHT: The Reporting Items for Practice Guidelines in Healthcare; AGREE II: Appraisal of Guidelines for Research and Evaluation II; IOM: Institute of Medicine; CheckUp: The Checklist for the Reporting of Updated Guidelines; TENS: Transcutaneous Electric Nerve Stimulation; RCTs: Randomized Controlled Trials; FDA: Food and Drug Administration; NASEM: The National Academies of Sciences, Engineering, and Medicine: AAMA: The American Academy of Medical Acupuncture; ACA: Affordable Care Act; BAcC: The British Acupuncture Council.

\section{Supplementary Information}

The online version contains supplementary material available at https://doi. org/10.1186/s13020-021-00558-4.

Additional file 1: Appendix S1. The professional field of the steering committee and the expert consensus group. Appendix S2. Details of panel members' declarations of interests. Appendix S3. Grading the certainty of evidence and the strength of recommendations. Appendix S4. Details of patients preference and values. Appendix S5. Details of expert consensus. Appendix S6. Summary of findings table.

\section{Acknowledgements}

We thank two patients (Runsen He and Zuodi Pan) who offer support during the development of guideline. We also thank the team of Guangdong Provincial Hospital of Chinese Medicine (Lihong Yang, Shaonan Liu, Xueling Chen, Xueyi Deng, Fuqin Kang) and Lanzhou University (Jieyun Li, Liangying Hou, Qian Zhang, Qi Wang, and Honghao Lai) for conducting evidence retrieval and literature screening to identify the potential randomized trials.

Contributors:

Steering committee: Charlie Changli Xue (School of Health and Biomedical Sciences, RMIT University), Haibo Zhang (Guangdong Provincial Hospital of Chinese Medicine), Xinfeng Guo (Guangdong Provincial Hospital of Chinese Medicine), Darong Wu (Guangdong Provincial Hospital of Chinese Medicine), Kehu Yang (Evidence-Based Medicine Centre, Lanzhou University; Chinese GRADE Centre) and Yaolong Chen (Evidence-Based Medicine Centre, Lanzhou University; Chinese GRADE Centre).

Clinical Co-chair: Charlie Changli Xue (School of Health and Biomedical Sciences, RMIT University), and Haibo Zhang (Guangdong Provincial Hospital of Chinese Medicine).

Method Co-chair: Kehu Yang (Evidence-Based Medicine Centre, Lanzhou University; Chinese GRADE Centre), Yaolong Chen (Evidence-Based Medicine Centre, Lanzhou University; Chinese GRADE Centre), Xinfeng Guo (Guangdong Provincial Hospital of Chinese Medicine), and Long Ge (School of Public Health, Lanzhou University). 
Members of expert consensus group: (in alphabetical order): Runsen $\mathrm{He}$ (Patient Partners), Haiqing Hua (Onclcogy Department of Bayi Hospital, Nanjing University of Chinese Medicine), Jinchang Huang (The Third Affiliated Hospital, Beijing University of Chinese Medicine), Ka-Kit Hui (Center for EastWest Medicine, Department of Medicine, David Geffen School of Medicine, University of California, Los Angeles), Fanrong Liang (Chengdu University of Traditional Chinese Medicine), Zuodi Pan (Patient Partners), Linpeng Wang (Beijing Hospital of Traditional Chinese Medicine, Capital Medical University), Darong Wu (Guangdong Provincial Hospital of Chinese Medicine), Bin Xu (Key Laboratory of Acupuncture and Medicine Research of Ministry of Education, Nanjing University of Chinese Medicine), Nenggui Xu (Guangzhou University of Chinese Medicine), Yufei Yang (Department of Oncology, Xiyuan Hospital, China Academy of Chinese Medical Sciences), Anthony Lin Zhang (School of Health and Biomedical Sciences, RMIT University), Weimin Zhang (Department of Oncology, Southern Theater Command General Hospital of PLA), Baixiao Zhao (Beijing University of Chinese Medicine), Bing Zhu (Institute of acupuncture and Moxibustion, China Academy of Chinese Medicine Sciences). Academic secretary group: Qi Wang (School of Public Health, Lanzhou University), Yihan He (Guangdong Provincial Hospital of Chinese Medicine). Members of evidence evaluation group: Long Ge (School of Public Health, Lanzhou University), Qi Zhou (Evidence-Based Medicine Centre, Lanzhou University), Lihong Yang (Guangdong Provincial Hospital of Chinese Medicine), Shaonan Liu (Guangdong Provincial Hospital of Chinese Medicine), Qi Wang (School of Public Health, Lanzhou University), Yihan He (Guangdong Provincial Hospital of Chinese Medicine), Jieyun Li (Evidence-Based Medicine Centre, Lanzhou University), Liangying Hou (School of Public Health, Lanzhou University), Qian Zhang (School of Nursing, Lanzhou University), Honghao Lai (School of Public Health, Lanzhou University), Qi Wang (School of Public Health, Lanzhou University), Xueling Chen (Guangdong Provincial Hospital of Chinese Medicine), Xueyi Deng (Guangdong Provincial Hospital of Chinese Medicine), Fugin Kang (Guangdong Provincial Hospital of Chinese Medicine). Contributors in drafting the guideline: Qi Wang (School of Public Health, Lanzhou University), Long Ge (School of Public Health, Lanzhou University), Yihan He (Guangdong Provincial Hospital of Chinese Medicine).

Contributors to the visual summary of recommendations: Long Ge (School of Public Health, Lanzhou University), Honghao Lai (School of Public Health, Lanzhou University), Qi Wang (School of Public Health, Lanzhou University).

\section{Authors' contributions}

$\mathrm{LG}, \mathrm{QW}$, and $\mathrm{YHH}$ drafted the manuscript. QW and $\mathrm{YHH}$ searched the literature and conducted the assessment. DRW, QZ, NGX, KHY, YLC, ALZ, HQH, JCH, KKH, FRL, LPW, BX, YFY, WMZ, BXZ, BZ gave suggestions and revised the manuscript. LG designed the study. CCX, XFG, and HBZ gave advice and revised the manuscript. All authors read and approved the final manuscript.

\section{Funding}

This guideline was funded by the National Key R\&D Program, China National Centre for Biotechnology Development (No. 2019YFC1709805). The funding is mainly used to pay for travel expenses and expert consultation expenses in the process of preparing the guideline.

\section{Availability of data and materials}

All data generated or analyzed during this study are included in Additional files.

\section{Declarations}

\section{Ethics approval and consent to participate}

Not applicable.

\section{Consent for publication}

Not applicable.

\section{Competing interests}

The guideline development was initiated by the international Trustworthy traditional Chinese Medicine Recommendations (TCM Recs) Working Group. Guangdong Provincial Hospital of Chinese Medicine and Evidence-Based Medicine Centre of Lanzhou University led the guideline development following the universal methodology by reaching the final consensus based on the latest research evidence and multidisciplinary expert opinions. All guideline panel members have completed the TCM Recs interest disclosure form. The TCM Recs judged that no panel member had any financial conflict of interest. Professional and academic interests are minimized as much as possible. Besides, we declare that the guideline will not be disseminated for commercial promotions.

\section{Author details}

${ }^{1}$ Department of Social Medicine and Health Management, School of Public Health, Lanzhou University, Lanzhou, China. ${ }^{2}$ Evidence Based Social Science Research Centre, School of Public Health, Lanzhou University, Lanzhou, China. ${ }^{3}$ WHO Collaborating Center for Guideline Implementation and Knowledge Translation, Lanzhou, China. ${ }^{4}$ Chinese GRADE Centre, Lanzhou University, Lanzhou, China. ${ }^{5}$ Guangdong Provincial Hospital of Chinese Medicine, Guangzhou, China. ${ }^{6}$ The Second Affiliated Hospital of Guangzhou University of Chinese Medicine, Guangzhou, China. ${ }^{7}$ Guangdong Provincial Academy of Chinese Medical Sciences, Guangzhou, China. ${ }^{8}$ Evidence-Based Medicine Center, School of Basic Medical Sciences, Lanzhou University, Lanzhou, China. ${ }^{9}$ South China Research Center for Acupuncture and Moxibustion, Medical College of Acu-Moxi and Rehabilitation, Guangzhou University of Chinese Medicine, Guangzhou, China. ${ }^{10}$ Key Laboratory of Evidence Based Medicine and Knowledge Translation of Gansu Province, Lanzhou, China. ${ }^{11}$ China-Australia International Research Centre for Chinese Medicine, School of Health and Biomedical Sciences, RMIT University, Melbourne, Victoria, Australia. ${ }^{12}$ Oncology Department of Bayi Hospital, Nanjing University of Chinese Medicine, Nanjing, China. ${ }^{13}$ The Third Affiliated Hospital, Beijing University of Chinese Medicine, Beijing, China. ${ }^{14}$ Center for East-West Medicine, Department of Medicine, David Geffen School of Medicine, University of California, Los Angeles, Santa Monica, USA. ${ }^{15}$ Chengdu University of Traditional Chinese Medicine, Chengdu, China. ${ }^{16}$ Beijing Hospital of Traditional Chinese Medicine, Capital Medical University, Beijing, China. ${ }^{17}$ Key Laboratory of Acupuncture and Medicine Research of Ministry of Education, Nanjing University of Chinese Medicine, Nanjing, China. ${ }^{18}$ Department of Oncology, Xiyuan Hospital, China Academy of Chinese Medical Sciences, Beijing, China. ${ }^{19}$ Department of Oncology, Southern Theater Command General Hospital of PLA, Guangzhou, China. ${ }^{20}$ School of Traditional Chinese Medicine, Beijing University of Chinese Medicine, Beijing, China. ${ }^{21}$ Institute of Acupuncture and Moxibustion, China Academy of Chinese Medicine Sciences, Beijing, China.

Received: 29 October 2021 Accepted: 15 December 2021 Published online: 05 January 2022

\section{References}

1. World Health Organization. Cancer. https://www.who.int/news-room/ fact-sheets/detail/cancer. 2018.

2. World Health Organization. Latest global cancer data: cancer burden rises to 19.3 million new cases and 10.0 million cancer deaths in 2020. http:// www.mbsonline.gov.au/internet/mbsonline/publishing.nsf/Content/ 432EE55FAB58E5C4CA257D6B001AFB8A/\%24File/201411-MBS.pdf. 2020.

3. Laird B, Colvin L, Fallon M. Management of cancer pain: basic principles and neuropathic cancer pain. Eur J Cancer. 2008;44(8):1078-82.

4. Portenoy RK. Treatment of cancer pain. Lancet. 2011;377(9784):2236-47.

5. World Health Organization. WHO Guidelines for the pharmacological and radiotherapeutic management of cancer pain in adults and adolescents. https://www.who.int/publications/i/item/who-guidelines-for-the-pharm acological-and-radiotherapeutic-management-of-cancer-pain-in-adultsand-adolescents. 2019.

6. Sharfstein JM, Olsen Y. Lessons learned from the opioid epidemic. JAMA. 2019;322(9):809-10.

7. Lyman GH, Greenlee H, Bohlke K, Bao T, DeMichele AM, Deng GE, et al. Integrative therapies during and after breast cancer treatment: ASCO endorsement of the SIO clinical practice guideline. J Clin Oncol. 2018;36(25):2647-55.

8. Vickers AJ, Vertosick EA, Lewith G, MacPherson H, Foster NE, Sherman KJ, et al. Acupuncture for chronic pain: update of an individual patient data meta-analysis. J Pain. 2018;19(5):455-74.

9. He YH, Guo XF, May BH, Zhang AL, Liu LH, Lu CJ, et al. Clinical evidence for association of acupuncture and acupressure with improved cancer pain: a systematic review and meta-analysis. JAMA Oncol. 2020;6(2):271-8. 
10. Mao JJ, Palmer CS, Healy KE, Desai K, Amsterdam J. Complementary and alternative medicine use among cancer survivors: a population-based study. J Cancer Surviv. 2011;5(1):8-17.

11. Ma BY, Zhu SJ, Lu DR, Chen F, Lu DX. Acupuncture and moxibustion treating cancer pain: a review of domestic and overseas clinical researches in recent five years. J Clin Acupunct-Mox. 2019;35(7):83-8.

12. Swarm RA, Paice JA, Anghelescu DL, Are M, Bruce JY, Buga S, et al. Adult cancer pain, version 2.2021, NCCN clinical practice guidelines in oncology. J Natl Compr Canc Netw. 2021;19(3):329-59.

13. Wengstrom Y, Geerling J, Rustoen T. European oncology nursing society breakthrough cancer pain guidelines. Eur J Oncol Nurs. 2014;18(2):127-31.

14. Liou KT, Mao JJ. Moving the needle: promoting the research, dissemination, and implementation of oncology acupuncture. J Altern Complement Med. 2020;26(2):85-7.

15. Institute of Medicine. National Academy of Sciences. Practice guidelines we can trust. Washington, DC: National Academies Pr; 2011. www.natio nalacademies.org. 2019

16. Hsu CC, Sandford BA. The Delphi technique: making sense of consensus Pract Assess Res Eval. 2007;12(10):1-8.

17. Black N, Murphy M, Lamping D, McKee M, Sanderson C, Askham J, et al. Consensus development methods: a review of best practice in creating clinical guidelines. J Health Serv Res Po. 1999;4(4):236-48.

18. World Health Organization. WHO handbook for guideline development, 2nd edition. https://apps.who.int/medicinedocs/en/m/abstract/Js220 83en/. 2014.

19. Chen Y, Yang K, Marusic A, Qaseem A, Meerpohl JJ, Flottorp S, et al. A reporting tool for practice guidelines in health care: the RIGHT statement. Ann Intern Med. 2017;166(2):128-32.

20. Brouwers MC, Kho ME, Browman GP, Burgers JS, Cluzeau F, Feder G, et al. AGREE II: advancing guideline development, reporting and evaluation in health care. J Clin Epidemiol. 2010;63(12):1308-11.

21. Guyatt GH, Oxman AD, Vist GE, Kunz R, Falck-Ytter Y, Alonso-Coello P, et al. GRADE: an emerging consensus on rating quality of evidence and strength of recommendations. BMJ. 2008;336(7650):924-6.

22. Schünemann H, Brożek J, Guyatt GH, Oxman AD. GRADE handbook for grading quality of evidence and strength of recommendations. Updated October 2013. The GRADE Working Group. 2013. https://www.guidelined evelopment.org/handbook. Accessed May 142020.

23. Dworkin RH, Turk DC, McDermott MP, Peirce-Sandner S, Burke LB, Cowan $\mathrm{P}$, et al. Interpreting the clinical importance of group differences in chronic pain clinical trials: IMMPACT recommendations. Pain. 2009;146(3):238-44

24. He YH, Xu NG, Zhang HB, Xue CL, Kang FQ, Wang Q, et al. Acupoint selection for cancer pain: based on current evidence and Delphi method. Chin Acupunct-Mox. 2021;41(10):1161-5.

25. Zhang Y, Alonso-Coello P, Guyatt GH, Yepes-Nunez JJ, Akl EA, Hazlewood $G$, et al. GRADE guidelines: 19. Assessing the certainty of evidence in the importance of outcomes or values and preferences-risk of bias and indirectness. J Clin Epidemiol. 2019;111:94-104.

26. Critical Appraisal Skills Programme. CASP qualitative checklist. https:// casp-uk.net/casp-tools-checklists/. 2019.

27. Vernooij RW, Alonso-Coello P, Brouwers M, Garcia LM, CheckUp Panel. Reporting items for updated clinical guidelines: checklist for the reporting of updated guidelines (CheckUp). PLoS Med. 2017;14(1): e1002207.

28. Alimi D, Rubino C, Pichard-Leandri E, Fermand-Brulé S, Dubreuil-Lemaire ML, Hill C. Analgesic effect of auricular acupuncture for cancer pain: a randomized, blinded, controlled trial. J Clin Oncol. 2003;21(22):4120-6.

29. Chen H, Liu TY, Kuai L, Zhu J, Wu CJ, Liu JM. Electroacupuncture treatment for pancreatic cancer pain: a randomized controlled trial. Pancreatology. 2013;13(6):594-7.

30. Crew KD, Capodice JL, Greenlee H, Brafman L, Fuentes D, Awad D, et al. Randomized, blinded, sham-controlled trial of acupuncture for the management of aromatase inhibitor-associated joint symptoms in women with early-stage breast cancer. J Clin Oncol. 2010;28(7):1154-60.

31. Hershman DL, Unger JM, Greenlee H, Capodice JL, Lew DL, Darke AK, et al. Effect of acupuncture vs sham acupuncture or waitlist control on joint pain related to aromatase inhibitors among women with early-stage breast cancer: a randomized clinical trial. JAMA. 2018;320(2):167-76.

32. Kim K, Lee S. Intradermal acupuncture along with analgesics for pain control in advanced cancer cases: a pilot, randomized, patient-assessor-blinded, controlled trial. Integr Cancer Ther. 2018;17(4):1137-43.

33. Mao JJ, Xie SX, Farrar JT, Stricker CT, Bowman MA, Bruner D, et al. A randomised trial of electro-acupuncture for arthralgia related to aromatase inhibitor use. Eur J Cancer. 2014;50(2):267-76.

34. Ruela LO, lunes DH, Nogueira DA, Stefanello J, Gradim CVC. Effectiveness of auricular acupuncture in the treatment of cancer pain: randomized clinical trial. Rev Esc Enferm USP. 2018;52: e03402.

35. Pfister DG, Cassileth BR, Deng GE, Yeung KS, Lee JS, Garrity D, et al. Acupuncture for pain and dysfunction after neck dissection: results of a randomized controlled trial. J Clin Oncol. 2010;28(15):2565-70.

36. Guo ZB, Guo GH, Yang JP, Shao Y. Effects of acupuncture on pain and quality of life for patients with advanced gastric cancer. Int J Chin Med. 2015;37(4):371-3.

37. Shen LF, Chen WY, Lv XD, Liu JL, Yang XM, Yao M, et al. Effects of electroacupuncture on improving sleep quality for patients with lung cancer pain. J Med Res. 2016;6:87-90

38. Wang Y. The clinical research of electro-acunpuncture therapy combined with oxycontin to treat the pain of advanced non-small cell lung cancer [doctoral thesis]. Zhejiang J Tradit Chin Med. 2017;9:684-5.

39. Wang J, Lu DR, Bi R, Shu XN. Clinical observation on 30 cases of moderate and severe cancer pain of bone metastasis treated by auricular acupressure. Yunnan J Tradit Chin Med Materia Medica. 2015;2:43-5.

40. Zhu LN, Wang RL, Zong H, Fan QX. Effects of auricular acupressure combined with oxycontin for malignant neuropathic pain. Chin J Phys Med Rehabil. 2013;35(7):579-81.

41. Jiang KR. Clinical research on acupuncture on siguan points combined with auricular acupressure for cancer pain [doctoral thesis]. Guangzhou Univ Chin Med. 2011.

42. Liou KT, Trevino KM, Meghani SH, Li QS, Deng G, Korenstein D, et al. Fear of analgesic side effects predicts preference for acupuncture: a crosssectional study of cancer patients with pain in the USA. Support Care Cancer. 2021;29(1):427-35.

43. Bao T, Li SQ, Dearing JL, Piulson LA, Seluzicki CM, Sidlow R, et al. Acupuncture versus medication for pain management: a cross-sectional study of breast cancer survivors. Acupunct Med. 2018;36(2):80-7.

44. Enblom A. Patients' and physiotherapists' belief in and use of acupuncture for cancer-related symptoms. Acupunct Med. 2017;35(4):251-8.

45. Mallory MJ, Croghan KA, Sandhu NP, Lemaine V, Degnim AC, Bauer BA, et al. Acupuncture in the postoperative setting for breast cancer patients: a feasibility study. Am J Chin Med. 2015;43(1):45-56.

46. Mao JJ, Tan T, Li SQ, Meghani SH, Glanz K, Bruner D. Attitudes and barriers towards participation in an acupuncture trial among breast cancer patients: a survey study. BMC Complement Altern Med. 2014;14(7):1-9.

47. Frankel E, Garland S, Meghani SH, Vapiwala N, Mao JJ. Patients' perspectives on integrating acupuncture into the radiation oncology setting. Eur J Integr Med. 2014;6(5):532-7.

48. Garcia MK, Driver L, Haddad R, Lee R, Palmer JL, Wei Q, et al. Acupuncture for treatment of uncontrolled pain in cancer patients: a pragmatic pilot study. Integr Cancer Ther. 2014;13(2):133-40.

49. Johnstone PA, Polston GR, Niemtzow RC, Martin PJ. Integration of acupuncture into the oncology clinic. Palliat Med. 2002;16(3):235-9.

50. Weidenhammer W, Streng A, Linde K, Hoppe A, Melchart D. Acupuncture for chronic pain within the research program of 10 German Health Insurance Funds-basic results from an observational study. Complement Ther Med. 2007;15(4):238-46.

51. Lao LX, Hamilton GR, Fu JP, Berman BM. Is acupuncture safe? A systematic review of case reports. Altern Ther Health Med. 2003;9(1):72-83.

52. Lu W, Dean-Clower E, Doherty-Gilman A, Rosenthal DS. The value of acupuncture in cancer care. Hematol Oncol Clin N Am. 2008;22(4):631-48, viii.

53. Ambrosio EM, Bloor $\mathrm{K}$, MacPherson $\mathrm{H}$. Costs and consequences of acupuncture as a treatment for chronic pain: a systematic review of economic evaluations conducted alongside randomised controlled trials. Complement Ther Med. 2012;20(5):364-74.

54. Fan AY, Wang DD, Ouyang $\mathrm{H}$, Tian $\mathrm{H}$, Wei $\mathrm{H}$, He D, et al. Acupuncture price in forty-one metropolitan regions in the United States: an out-of-pocket cost analysis based on OkCopay.com. J Integr Med. 2019;17(5):315-20.

55. Fan AY, Miller DW, Bolash B, Bauer M, McDonald J, Faggert S, et al. Acupuncture's role in solving the opioid epidemic: evidence, costeffectiveness, and care availability for acupuncture as a primary, 
non-pharmacologic method for pain relief and management-white paper 2017. J Integr Med. 2017;15(6):411-25.

56. Badgut. Acupuncture: regulation \& coverage in Canada. http://www. activetcm.com/wp-content/uploads/2013/03/Badgut.org-AcupunctureRegulation-and-Coverage-Badgut.pdf. 2013.

57. Australian Government Department of Health. Medicare benefits schedule book. http://www.mbsonline.gov.au/internet/mbsonline/publishing. nsf/Content/432EE55FAB58E5C4CA257D6B001AFB8A/\%24File/201411MBS.pdf. 2014:11-51.

58. Jiang YG. TCM treatment in Switzerland is supported by medical insurance. Chin J Tradit Chin Med. 2015;3.

59. Li SL. Survey of development of Chinese acupuncture and moxibustion in Switzerland. Chin Acupunct-Mox. 2004;24(4):283-5.

60. Beijing Youth Daily. Acupuncture and massage will be included in the new rural cooperative medical care system. Women's Life. 2013;1:32.

61. AAMA. American Academy of Medical Acupuncture. http://www.medic alacupuncture.org. 2017.

62. Fan AY. "Obamacare" covers fifty-four million Americans for acupuncture as essential healthcare benefit. J Integr Med. 2014;12(4):390-3.

63. Chinese Medicine Board Ahpra. 2019/20 annual summary. https://www. chinesemedicineboard.gov.au/News/Annual-report.aspx. 2020.

64. Filshie J. Acupuncture for malignant pain. Acupunct Med. 1984;2:12-4.

65. Filshie J. Safety aspects of acupuncture in palliative care. Acupunct Med. 2001;19:117-22

66. Filshie J, Hester J. Guidelines for providing acupuncture treatment for cancer patients - a peer-reviewed sample policy document. Acupunct Med. 2006;24:172-82.

67. Council. BA. Acupuncture practitioners in the UK. https://www.acupu ncture.org.uk/public-content/about-the-bacc/4115-acupuncture-pract itioners-in-the-uk.html. 2021.

68. Lopez G, Lacey J, Christie AJ, Powers-James C, Narayanan S, Liu W, et al. Patient-reported outcomes in integrative oncology: bridging clinical care with research. Cancer J. 2019;25(5):311-5.

69. Ben-Arye E, Elly D, Samuels N, Gressel O, Shulman K, Schiff E, et al. Effects of a patient-tailored integrative oncology intervention in the relief of pain in palliative and supportive cancer care. J Cancer Res Clin Oncol. 2021. https://doi.org/10.1007/s00432-020-03506-1.

70. Carreira H, Williams R, Dempsey H, Stanway S, Smeeth L, Bhaskaran K. Quality of life and mental health in breast cancer survivors compared with non-cancer controls: a study of patient-reported outcomes in the United Kingdom. J Cancer Surviv. 2020. https://doi.org/10.1007/ s11764-020-00950-3.

71. Lopez G, Mao JJ, Cohen L. Integrative oncology. Med Clin N Am. 2017;101(5):977-85.

72. Deng G, Cassileth BR. Integrative oncology: complementary therapies for pain, anxiety, and mood disturbance. CA Cancer J Clin. 2005;55(2):109-16

73. Huang T, Hung T, Kim SI, Hui KK. Acupuncture for management of symptom clusters in a patient with metastatic breast cancer: a case report. OBM Integr Complement Med. 2020;5(1):1-6.

74. Li D, Sun RR, Li QL, Ma Q, Zeng YL, Jia XZ, et al. Acupuncture combined with opioid drugs on moderate and severe cancer pain: a randomized controlled trial. Zhongguo Zhen Jiu. 2020;40(3):257-61.

\section{Publisher's Note}

Springer Nature remains neutral with regard to jurisdictional claims in published maps and institutional affiliations.

Ready to submit your research? Choose BMC and benefit from:

- fast, convenient online submission

- thorough peer review by experienced researchers in your field

- rapid publication on acceptance

- support for research data, including large and complex data types

- gold Open Access which fosters wider collaboration and increased citations

- maximum visibility for your research: over $100 \mathrm{M}$ website views per year

At BMC, research is always in progress.

Learn more biomedcentral.com/submissions 